\title{
Maternal separation followed by isolation-housing differentially affects prepulse inhibition of the acoustic startle response in C57BL/6 mice
}

\begin{tabular}{|r|l|}
\hline Journal: & Developmental Psychobiology \\
\hline Manuscript ID & DEV-15-125.R1 \\
\hline Wiley - Manuscript type: & Research Article \\
\hline Date Submitted by the Author: & n/a \\
\hline Complete List of Authors: & $\begin{array}{l}\text { Bailoo, Jeremy; The University of Bern, Switzerland, Division of Animal } \\
\text { Welfare } \\
\text { Varholick, Justin; University of Bern, Division of Animal Welfare } \\
\text { Garza, Xavier; The University of North Carolina at Greensboro, Psychology } \\
\text { Jordan, Richard; The University of North Carolina at Greensboro, } \\
\text { Psychology } \\
\text { Hintze, Sara; The University of Bern, Switzerland, Division of Animal } \\
\text { Welfare }\end{array}$ \\
\hline Keywords: & $\begin{array}{l}\text { maternal separation, early handling, match/mismatch hypothesis, } \\
\text { isolation-housing, prepulse inhibition }\end{array}$ \\
\hline
\end{tabular}

\section{SCHOLARONE ${ }^{m}$}

Manuscripts 
1 Maternal separation followed by isolation-housing differentially affects prepulse inhibition of the acoustic startle response in C57BL/6 mice.

3

4

5

6

7

8

9 Jeremy D. Bailoo ${ }^{1}$, Justin A. Varholick ${ }^{1}$, Xavier J. Garza ${ }^{2}$, Richard L. Jordan ${ }^{2}$ and Sara Hintze ${ }^{1}$

10

$11{ }^{1}$ Division of Animal Welfare, The University of Bern, Bern, Switzerland, 3012, ${ }^{2}$ Department of

12 Psychology, The University of North Carolina-Greensboro, Greensboro, NC 27412.

13

14

15

16

17 Short title: MATERNAL SEPARATION AFFECTS PREPULSE INHIBITON

18 Corresponding Author:

19 Jeremy D. Bailoo, Ph.D.

20 Division of Animal Welfare

21 The University of Bern,

22 Bern, Switzerland 3012

23 Ph: 919-308-9069

24 jeremy.bailoo@vetsuisse.unibe.ch 
25 The current study examined the effect of early-life stress in C57BL/6 offspring reared 26 under four conditions: typical animal facility rearing (AFR, Control), early handling (EH, daily

2715 min. separation from dam), maternal separation (MS, daily $4 \mathrm{hr}$. separation from dam), and

28 maternal and peer separation (MPS, daily $4 \mathrm{hr}$. separation from dam and from littermates). After

29 weaning, mice across these four conditions were either housed socially (2 - 3/cage) or in

30 isolation (1/cage) and then tested for prepulse inhibition in adulthood. Isolation-housed MPS

31 subjects displayed greater deficits in prepulse inhibition relative to socially-housed MPS subjects

32 while socially-housed AFR subjects displayed greater deficits in prepulse inhibition relative to

33 isolation-housed AFR subjects. The results indicate that these treatment conditions represent a

34 potentially valuable model for evaluating the match/mismatch hypothesis in regards to

35 neuropsychiatric dysfunction.

36

37 Keywords: maternal separation, early handling, match/mismatch hypothesis, isolation-housing, 38 prepulse inhibition 
39 Exposure to chronic stress during development has been associated with an increased risk

40 for neuropsychiatric dysfunction in later life (de Kloet, Joëls, \& Holsboer, 2005). Two primary

41 and seemingly contradictory hypotheses have been used in the evaluation of such risk; the

42 cumulative stress hypothesis and the match/mismatch hypothesis. The more traditional of these

43 hypotheses, the cumulative stress hypothesis, states that exposure to consecutive stressors across

44 development increases allostatic load, vulnerability to aversive challenges, and susceptibility to

45 neuropsychiatric dysfunction in later life (McEwen, 2003). Conversely, the match/mismatch

46 hypothesis states that an individual who has experienced high levels of stress early in

47 development is better able to cope with stressors later in life compared to an individual who has

48 experienced no or low levels of early-life stress, and therefore, is at a decreased risk for

49 neuropsychiatric dysfunction (Schmidt, 2011).

50 Rodents have a long history of use in modeling such neuropsychiatric risks (Pryce,

51 Rüedi-Bettschen, Dettling, \& Feldon, 2002) and although data from such models are not always

52 consistent (Lehmann \& Feldon, 2000), one model that is generally thought to be predictive of

53 vulnerable phenotypes is maternal separation (Branchi \& Cirulli, 2014). Brief periods ( 15

54 minutes) of dam-pup separation (i.e., early handling, EH) may lead to offspring exhibiting

55 decreased reactivity of the hypothalamic pituitary adrenal (HPA) axis to stress-inducing

56 situations in later adult life (c.f., Kaffman \& Meaney, 2007). However, this result is only

57 observed if the comparison group is not handled until weaning (Levine, 2002). If the comparison

58 group is a typical animal facility reared (AFR) group, no differences in stress reactivity are

59 observed between these groups (Levine, 2002). 
Longer periods of dam-pup separation (between 3 - 6 hours), conversely, produce an

61 exaggerated HPA axis response to a stressor in later adult life (Meaney, 2001). The two most

62 common forms of these longer periods of separation are maternal separation (MS) of the dam

63 from the pups, and maternal and peer separation (MPS) of the dam from the pups in addition to

64 the littermates from one another. The effects of MS/MPS on HPA axis reactivity to stressors are

65 not consistent throughout the literature. Such inconsistencies are thought to be a consequence of

66 methodological differences between laboratories, including but not limited to the timing,

67 duration, and number of MS/MPS episodes (for review, c.f., Millstein \& Holmes, 2007).

68 Both EH and MS/MPS lead to increases in maternal care for approximately 1 - 2 hours

69 after reuniting the pups with the dam (Liu et al., 1997; Macrí, Mason, \& Würbel, 2004). It is this

70 increase in maternal behavior that has traditionally been associated with the series of

71 downstream effects that mediate the response of the pups to stressors as adults (Meaney, 2001;

72 Smotherman \& Bell, 1980), although some have challenged this premise (Macrì \& Würbel,

73 2006).

74 To date, only one study has included and systematically compared the effects of these

75 three most common treatment conditions (EH, MS, MPS) on maternal care and adult offspring

76 behavior in a single experiment (Bailoo, Jordan, Garza, \& Tyler, 2013). In this study, we

77 demonstrated that one consequence of MS/MPS is an increase in maternal care in the immediate

78 reunion phase relative to EH/AFR groups. Thus, while MS/MPS groups are generally thought to

79 be associated with poorer outcomes because the longer periods of separation deprive the pups of

80 maternal care (Caldji, Francis, Sharma, Plotsky, \& Meaney, 2000), the results of our original

81 study suggested that groups receiving the highest levels of maternal care were largely comprised 
82 of the MS/MPS groups and displayed decreased "anxiety-like behavior" in an open field

83 compared to groups that received lower levels of maternal care (largely comprised of AFR/EH 84 groups).

85 In the current study, we extended the results of our previous work by investigating the 86 development of an endophenotype related to neuropsychiatric dysfunction, prepulse inhibition of

87 the acoustic startle response (Geyer, Krebs-Thomson, Braff, \& Swerdlow, 2001). Prepulse

88 inhibition of the startle response is a neurological phenomenon, in which a weaker sensory

89 stimulus inhibits the reaction of an organism to a subsequent strong and typically startling

90 stimulus (a.k.a., sensorimotor gating) (Ison \& Hoffman, 1983). Disruption of prepulse inhibition

91 is noted in humans with symptoms of neuropsychiatric dysfunction and has previously been

92 modeled in mice using manipulations occurring at two points in development, pre-weaning

93 (maternal separation) and post-weaning (isolation-housing) (for review, c.f., Braff, Geyer, \&

94 Swerdlow, 2001).

95 Some studies have hypothesized that the application of both paradigms successively may

96 lead to potentiated deficits in prepulse inhibition in later adult life (Matsumoto et al., 2011;

97 Weiss, Domeney, Moreau, Russig, \& Feldon, 2001). However, no study to date has evaluated the

98 additive or interactive effects of the most common dam-pup separation paradigms (EH, MS,

99 MPS) in conjunction with post-weaning housing (social- vs. isolation-housing); a gap in the

100 literature which this study addresses. Additionally, an AFR control group was included, as this is

101 the most common reference group used in these investigations.

102 The successive application of these manipulations permits for the evaluation of both the 103 cumulative stress and the match/mismatch hypotheses. Specifically, if the match/mismatch 
104 hypothesis is supported, then based on the existing maternal behavior data (Bailoo et al., 2013),

105 it was predicted that MS/MPS subjects housed in social isolation post-weaning would show an

106 increased susceptibility for neuropsychiatric dysfunction (operationally defined here as

107 disruption of the startle response and prepulse inhibition of the startle response) while AFR/EH

108 subjects housed in social isolation post-weaning would show a decreased susceptibility for

109 neuropsychiatric dysfunction. Conversely, if the cumulative stress hypothesis is supported,

$110 \mathrm{AFR} / \mathrm{EH}$ subjects housed in social isolation post-weaning would show the greatest susceptibility

111 for neuropsychiatric dysfunction.

112

113 Method

114 General Husbandry Procedures

115 Subjects were housed in $29 \times 19 \times 12 \mathrm{~cm}$ polypropylene cages on a 14:10 light/dark cycle

116 with lights on at $14: 00$. Temperature was maintained at $21^{\circ} \mathrm{C}$ and humidity at $50 \%$. Subjects

117 were provided with food and water ad libitum, nesting material, and Harlan Aspen Sani-Chips

118 bedding approximately $1.3 \mathrm{~cm}$ deep. Weekly cage changes occurred between 14:00 and 15:00.

\section{Breeding Subjects}

120 Ten female and five male C57BL/6 mice were purchased from Harlan Laboratories,

121 Frederick, MD, USA. Using a common breeding strategy, these ten females each produced three

122 litters. The first two litters were used for training purposes with students and for piloting a

123 behavioral test battery. Experimental subjects were produced by breeding different pairs of

124 animals from the third litter of animals and their offspring onwards. Breeding for at least three

125 generations was performed to reduce or remove experimental artifacts which may have arisen as 
126 a consequence of differential rearing, husbandry, and the laboratory environment at Harlan

127 Laboratories.

128 Experimental Subjects

129 Forty-four litters were bred across 11 cohorts and assigned via a pseudo-random manner

130 to one of the four groups described below. Forty-one of these litters were primiparous.

131 Assignment was such that there was always a cohort of litters representing each of the four

132 groups at any given time. The average litter size was six, with a minimum of four and a

133 maximum of eight offspring. One randomly selected male and female from each of the 44 litters

134 was used in another study (Bailoo et al., 2013). The remaining offspring of these litters were

135 used in this project (c.f., Table 1).

\section{Insert Table 1 Here}

\section{Maternal Separation Procedures}

138 Dam-offspring separations occurred from post-natal day (PND) 2 to 14 (day of birth was

139 PND 0) and were performed by the same two experimenters. First, the dam was removed from

140 the home-cage and placed into a clean cage with bedding. Then, pups were individually removed

141 from the home-cage and placed into a clean cage with bedding. After pup removal, the dam was

142 replaced into the home-cage for the duration of the separation.

143 MS pups were separated from the dam for 240 minutes (between 0900 and 1300), and

144 MPS pups were separated from both the dam and their littermates for 240 minutes (between

1450900 and 1300). Both MS and MPS pups were placed into a standard $(29 \times 19 \times 12 \mathrm{~cm})$

146 polypropylene cage. For the MPS group, frosted Plexiglas ${ }^{\circledR}$ partitions were placed within the

147 cage to create eight separate compartments, one for each pup (Millstein, Ralph, Yang, \& 
148 Holmes, 2006). This partition eliminated tactile and visual interactions between littermates.

149 Using infrared heating lamps, pup cages were maintained at $31^{\circ} \mathrm{C}\left( \pm 2^{\circ} \mathrm{C}\right)$ for the 240 minute

150 separation groups (MS and MPS) in order to prevent thermoregulatory distress. Pups in the EH

151 group were separated from the dam for 15 minutes in the same manner as the MS group

152 (between 12:45 and 13:00) but were not placed under heating lamps. All separation procedures

153 ended at 13:00, one hour before lights on.

154 For reunion, the dam was removed from the home-cage and temporarily placed into a 155 clean cage with bedding (the same cage used previously). Then, the pups followed by the dam 156 were replaced into the home-cage. An AFR control group was not separated from the dam but 157 received the same weekly cage changes as the other three groups.

158 Weekly cage changes began when the pups were seven days old (PND 7). The dam was 159 removed and placed in a clean cage with bedding. Some soiled bedding from the home-cage was 160 sprinkled into a new cage and the nest from the home-cage was relocated (same side/area) to this 161 new cage. Pups were then individually placed in the relocated nest. The dam was then placed in 162 the new home-cage. This process took less than one minute. Regular cage changes occurred on 163 PND 7 and 14 between 1400 and 1500 hours.

\section{Maternal Behavior}

165 The maternal behavior of the dams in each treatment group was characterized and has 166 been detailed elsewhere (Bailoo et al., 2013). Briefly, maternal behaviors were recorded for one 167 hour both before and after each separation period every other day from PND 2 to 14 during the 168 dark phase under a $50 \mathrm{~W}$ infrared lamp using a closed-circuit camera connected to a high 169 definition video recorder. All recordings for all groups occurred at the same time during the 
170 light/dark cycle. Maternal behaviors were scored using Noldus Observer 5.1 on an ethogram of 171 nursing postures and parental care behaviors adapted from Stern \& Johnson (1989) and Shoji \& 172 Kato (2006).

\section{Post-weaning Housing}

174 Subjects were weaned on PND 21. Animals that were not used in the original study

175 (Bailoo et al., 2013) were randomly allocated to either social-housing (2 - 3 subjects/cage with 176 their same sex, same group siblings) or isolation-housing (1 subject/cage) for the duration of the 177 experiment. When fewer than three extra animals per litter per sex were present, assignment to 178 social-housing was given priority. Cage changes continued to occur once per week thereafter.

179 Sensorimotor Gating Procedures

180 Startle response was measured using the SR-LAB (San Diego Instruments, San Diego, 181 CA, USA) startle response measurement system, including software (Paylor \& Crawley, 1997).

182 In this system, an acrylic cylinder (inner diameter $4 \mathrm{~cm}$, length $13 \mathrm{~cm}$ ) for holding the mouse 183 was mounted on a Plexiglas ${ }^{\circledR}$ platform with a piezoelectric accelerometer unit attached below the 184 acrylic cylinder. The piezoelectric unit transduced vibrations created by mouse body movements 185 into signals that were rectified and stored by a microcomputer and then converted into a signal 186 proportional to response amplitude. The acrylic cylinder and platform were located in a sound187 attenuated chamber with a loudspeaker located $33 \mathrm{~cm}$ above the cylinder and house-light.

188 Baseline values of startle between the two SR-LAB chambers used in this study were equated 189 using the SR-LAB Standardization Unit at the onset of the experiment.

190 Subjects were tested individually by one of the original experimenters in one of the two 191 chambers in a predefined pseudo-random manner between PND 60 - 70. Following a 5 minute 
192 acclimation period in the cylinder, individual subjects were presented 50 trials over a 12.39

193 minute session. Each session consisted of five different trial types presented in pseudo-random

194 order in 10 blocks. Three of the five trial types consisted of a 20 ms prepulse stimulus (72-, 76-,

195 84-dB white noise) presented so that the onset of the prepulse stimulus occurred $100 \mathrm{~ms}$ before

196 the onset of the $40 \mathrm{~ms}, 120-\mathrm{dB}$ white-noise startle stimulus. The fourth of the five trial types

197 involved the presentation of the startle stimulus alone, and the fifth trial type was background

198 only $(65-\mathrm{dB})$ to establish baseline movement in the test chamber. The average inter-trial interval

199 was 15 s ( 9 - 23 s range). The amplitude of the startle response was measured every $1 \mathrm{~ms}$ for 65

200 ms starting with the onset of the startle stimulus. When a startle stimulus (120-dB white noise on

201 a 65-dB white noise background) follows a prepulse, the amplitude of the startle response is

202 reduced, compared with its amplitude when the startle stimulus is presented without a prepulse.

203 This amplitude reduction is called prepulse inhibition (our primary outcome variable) and is the

204 percentage reduction of the mean startle amplitude for the prepulse trial expressed as the

205 percentage reduction of the mean startle amplitude for startle-alone trial:

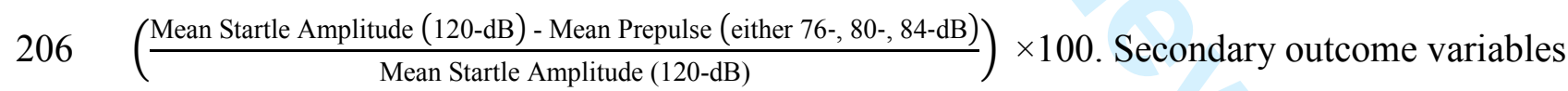

207 included baseline startle response amplitude at $68-\mathrm{dB}$ and acoustic startle response amplitude at $208 \quad 120-d B$

209

210 Statistical Analyses

211 All statistical analyses were performed with IBM SPSS Statistics (version 23) using the

212 MIXED procedure. Assumptions of normality of error distribution, homogeneity of variance, and

213 parameter linearity were examined graphically. No transformation of data was required based on 
214 these inspections. Predictors used in all models were sex (male, female), pre-weaning group 215 (AFR, EH, MS, MPS), post-weaning housing (socially-housed, isolation-housed), and decibel 216 level (respectively 68-, 76-, 80-, 84-, 120-dB, at level 1 to account for repeated measurement). 217 For all models built, 1) individual animals nested within litter, and 2) chambers were included as 218 random effects to accommodate for dependencies in the experimental design. Inclusion of these 219 random effects allowed us to partition the variation associated with each of these variables, and 220 to obtain a treatment effect estimate that was independent of these variables. Subject weight was 221 also included as a covariate (control factor) in the model, as the intensity of the startle response is 222 affected by body weight (Blaszczyk \& Tajchert, 1996). In all analyses, the full factorial model 223 was the best model (based on $\triangle \mathrm{AIC}$ and $\triangle \mathrm{BIC}$ ). P-values below 0.05 were considered 224 statistically significant, and significant main effects and interactions were probed with 225 Bonferroni corrected post hoc comparisons.

$227 \quad$ Results

228 Prepulse Inhibition of the Startle Response

229 A main effect of decibel level was observed, indicating that irrespective of sex, pre-

230 weaning group, and post-weaning housing, as prepulse intensity increased, prepulse inhibition of 231 the startle response also increased, $\left(F_{2,169}\right)=49.84, p=0.00001$ (Figure 1).

Insert Figure 1 here.

233 A main effect of sex was also observed, indicating that regardless of prepulse level, males 234 displayed lower levels of prepulse inhibition of the startle response compared to females, $235\left(F_{1,39}\right)=8.378, p=0.006$ (Figure 2). 
Insert Figure 2 here.

237 Lastly, an interaction between pre-weaning treatment condition and post-weaning 238 housing condition was observed, $\left(F_{1,39}\right)=8.378, p=0.005$ (Figure 3). Post hoc analyses 239 comparing post-weaning housing condition within pre-weaning group indicated that socially240 housed AFR subjects $(M=24.21, S E=3.80)$ displayed lower levels of prepulse inhibition 241 compared to isolation-housed AFR subjects $(M=39.72, S E=3.08)$. The inverse pattern of 242 results was observed between isolation-housed $(M=25.73, S E=3.19)$ and socially-housed $(M=$ $243 \quad 37.793, S E=3.205)$ MPS subjects.

Insert Figure 3 here.

245

246

247

\section{Baseline Startle Response Amplitude (68 dB)}

A main effect of pre-weaning group, $\left(F_{3,225}\right)=4.750, p=0.004$, and an interaction between pre-weaning group and post-weaning housing was observed, $\left(F_{3,225}\right)=3.101, p=0.031$. Post hoc analyses comparing post-weaning housing conditions within pre-weaning group yielded no significant differences between any of our groups. However, post hoc analyses comparing pre-weaning group within post-weaning housing condition indicated that isolation-housed MPS subjects $(M=32.25, S E=2.86)$ displayed a significantly higher baseline startle response amplitude compared to isolation-housed AFR subjects $(M=15.73, S E=3.24)$ (Figure 4). While isolation-housed MS subjects also displayed higher levels of baseline startle response amplitude relative to isolation-housed AFR subjects (Mean Difference $=10.70)$, this difference failed to reach statistical significance $(p=0.055)$.

\section{Insert Figure 4 here.}

\section{Startle Response Amplitude (120 dB)}


258 A main effect of $\operatorname{sex},\left(F_{1,133}\right)=12.20, p=0.001$, was observed, indicating that male mice 259 displayed a higher acoustic startle response amplitude, $(M=382.13, S E=20.46)$, than female 260 mice, $(M=285.44, S E=18.64)($ Figure 5).

Insert Figure 5 here.

262 A main effect of post-weaning housing was also observed, $\left(F_{1,133}\right)=3.926, p=0.049$, 263 indicating that isolation-housed subjects displayed a lower amplitude of the acoustic startle 264 response, $(M=306.36, S E=2.49)$, relative to socially-housed animals, $(M=361.21, S E=$ 265 16.14) (Figure 6).

\section{Insert Figure 6 here.}

268 Discussion

269 The overall aim of this study was to assess the additive (cumulative stress hypothesis) or 270 interactive effects (match/mismatch hypothesis) of early-life experiences in the form of dam271 offspring separation and subsequent post-weaning social housing on the manifestation of adult 272 prepulse inhibition using the C57BL/6 inbred mouse. Analysis of the primary outcome variable, 273 prepulse inhibition of the acoustic startle response, provided direct evidence for the

274 match/mismatch hypothesis. Specifically, isolation-housed MPS subjects displayed a deficit in 275 prepulse inhibition relative to socially-housed MPS subjects while socially-housed AFR subjects 276 displayed a deficit in prepulse inhibition relative to isolation-housed AFR subjects.

\section{Maternal Behavior during the pre-weaning phase}

278 In the earlier experiment (Bailoo et al., 2013), we reported that the effects of the pre279 weaning manipulations were restricted to the reunion phase with the dam, with an overall 
280 increase in maternal behavior for the longer separated groups (MS \& MPS). Moreover, for the 281 MS/MPS groups, the homeostatic balance of the pups was maintained using heat lamps $(31 \pm$ $2822^{\circ} \mathrm{C}$ ), no indication of food deprivation was present, and correspondingly, higher weaning 283 weights were observed. Thus, subjects in the MS/MPS groups experienced a better outcome, at 284 least in regards to levels of maternal care, as a consequence of these manipulations relative to 285 AFR/EH groups.

286 Effects of pre-weaning group and post-weaning housing on prepulse inhibition

287 Isolation-housed AFR subjects displayed higher levels of prepulse inhibition relative to 288 socially-housed AFR subjects, with the opposite relation observed for MPS subjects; a result that

289 is consistent with the match/mismatch hypothesis. This result is most likely because AFR

290 subjects received lower levels of maternal care pre-weaning when compared to MPS groups,

291 which has been associated with a stressful early environment and poorer adult outcomes in

292 rodents (Champagne et al., 2008).Thus, in this study, AFR subjects experienced a "match" when

293 housed in isolation while MPS subjects experienced a "mismatch" when housed in the same 294 manner.

295 A main effect of decibel level was observed, indicating that as the intensity of the 296 prepulse increased, inhibition of the startle response correspondingly increased. This result 297 demonstrated that the prepulse inhibition experimental procedure used in this study was 298 effective.

299 A main effect of sex was also observed, with males displaying lower levels of prepulse 300 inhibition relative to females, after correcting for body weight. This result was surprising given 301 that the literature supports the contention of a sex difference, but in the opposite direction (Braff 
302 et al., 2001). However, in many of the studies investigating or reporting sex differences, prepulse

303 inhibition of startle is generally confounded by body weight (Blaszczyk \& Tajchert, 1996).

304 Specifically, male rodents generally weigh more than females, have greater muscle mass and

305 associated motor strength, and relatedly, display a greater startle response and a deficiency in the

306 ability to display prepulse inhibition. In the few studies that we are aware of that statistically

307 corrected for this sex/weight correlation, this difference disappears or at least is less clear in

308 regards to the direction of this effect (e.g., Blaszczyk \& Tajchert, 1996). Moreover, it is

309 important to note that this purported sex difference can be modulated by several other factors

310 including, for example, female hormonal state (c.f., Braff et al., 2001, for review). Thus,

311 explanation for this difference remains speculative at best and further work replicating this effect

312 and detailing the neurobiological mechanism is needed.

313 Effects of pre-weaning group and post-weaning housing on baseline startle (68-dB)

314 A significant interaction between pre-weaning group and post-weaning housing was

315 observed. However, probing this interaction in relation to our experimental question by

316 comparing the effects of either social- or isolation-housing within pre-weaning treatment groups

317 yielded no significant differences.

318 Effects of pre-weaning group and post-weaning housing on the startle response (120 dB)

319 An effect of post-weaning housing condition on acoustic startle response amplitude was

320 observed, with isolation-housed subjects displaying lower levels of startle relative to socially-

321 housed subjects. While this result is generally consistent with the literature, it should be noted

322 that Geyer and colleagues, in a systematic review (2001), have stated that while some studies

323 report an increase in acoustic startle response amplitude as a consequence of isolation-housing, 
324 others report no or the opposite effect. Therefore, acoustic startle response amplitude seems to be

325 the least predictive of neuropsychiatric dysfunction, at least in regards to whether corresponding

326 deficits in prepulse inhibition are observed (Varty, Braff, \& Geyer, 1999; Varty \& Geyer, 1998).

327 This may also be true of the data in our study, with deficits in startle responding observed

328 between the isolation- and socially-housed groups, but not in relation to pre-weaning treatment

329 conditions.

330 A main effect of sex on acoustic startle response amplitude was observed, with male mice

331 displaying a greater startle response than females. However, as noted above, further experimental

332 work is needed to replicate and delineate this effect.

\section{Limitations}

334 It is important to note that this study made use of "extra" animals from litters that had 335 been produced for use in a different study (Bailoo et al., 2013), and thus a fully balanced design

336 was not achieved. However, with the exception of the isolation-housed EH group, and given the

337 relatively large observed effect sizes, it may be argued that this experiment was sufficiently

338 powered and that these data are reliable. Moreover, given that the literature suggests that the

339 AFR condition is generally similar in phenotype to the EH condition, and that the maternal care

340 data recorded in this study supports this "homology", it can be speculated that the observed

341 differences with the isolation-housed AFR group are also applicable to the isolation-housed EH

342 group (Levine, 2002).

343 The pre-weaning manipulations and their effects on maternal care were described

344 previously (Bailoo et al., 2013). In those data, many aspects of maternal care were elevated but

345 those differences were restricted to the reunion phase in the longer separated groups (MS and 
346 MPS). It was hypothesized that since maternal care has been shown to mediate the relation

347 between these early experience paradigms and later offspring outcome, these groups would

348 exhibit the smallest deficits in prepulse inhibition. However, in this study, we observed this

349 relation only in the MPS and not in the MS group.

350 Several factors may account for the lack of an observed effect in our MS group. While it

351 is presumed that maternal care mediates the relation of pre-weaning separations to adult

352 phenotypes, including prepulse inhibition, perhaps other unmeasured factors might also affect

353 this relation (c.f., Macrì \& Würbel, 2006). For example, systematic work evaluating food

354 deprivation, thermoregulation, ultrasonic vocalization production, and behavioral changes and

355 adaptations by the pups (and their influence on the dam) as a consequence of these pre-weaning

356 manipulations remains largely unexamined. Without systematically acquiring such information,

357 it is unknown whether MS and MPS groups are equivalent. Only the levels of maternal care

358 exhibited to the pups upon reunion are similar. Future studies characterizing the differences

359 between the MS and MPS groups are therefore needed.

\section{Conclusion}

361 Deficits in prepulse inhibition are noted in humans with symptoms of neuropsychiatric

362 dysfunction such as schizophrenia, obsessive compulsive disorder, and attention deficit

363 hyperactivity disorder (Braff et al., 2001). Considerable evidence supports a high degree of

364 similarity between measures of prepulse inhibition in rodents and humans (e.g., Braff et al.,

365 2001; Ellenbroek, Geyer, \& Cools, 1995). Moreover, prepulse inhibition appears to be highly

366 conserved among vertebrates and is one of the few paradigms in which humans and animals are

367 tested in a similar fashion. Thus, investigation into the disruption of prepulse inhibition as a 
368 consequence of early experiences associated with an increased susceptibility for neuropsychiatric

369 dysfunction is well suited to rodent models (Swerdlow, Weber, Qu, Light, \& Braff, 2008).

370 This study was designed to investigate the additive effects of typical stress-related

371 manipulations applied at two different developmental periods, and extends previous work

372 employing these manipulations. Analysis of the primary outcome variable of this study, prepulse

373 inhibition, provides support for the match/mismatch hypothesis. Generally speaking, isolation-

374 housing should lead to deficits in prepulse inhibition. However, in this study, AFR subjects that

375 experienced the lowest levels of maternal care displayed deficits in prepulse inhibition when

376 housed socially compared to isolation. Conversely, MPS subjects that experienced high levels of

377 maternal care and were later housed in isolation displayed greater deficits in prepulse inhibition

378 compared to MPS subjects housed socially.

379 Future studies employing these early experience paradigms consecutively in the

380 evaluation of adult prepulse inhibition should benefit from our results. Specifically, if isolation-

381 housing is used, then robust differences can be observed simply between the AFR control

382 groups, with the noteworthy difference being that social housing leads to deficits in prepulse

383 inhibition relative to isolation-housing, at least in C57BL/6 mice. If the intention is to

384 analogously model the match/mismatch hypothesis (also termed "differential susceptibility" in

385 human research), then both the AFR and the MPS groups in social- and isolation-housing,

386 respectively, can be used to model this relation. 


\section{Notes}

The authors gratefully acknowledge the assistance of Dr. Amber N. Tyler, Dr. Douglas L. Wahlsten, Dr. Walter L. Salinger, Dr. Amrika Deonarine, Dr. Claudio L. Ferre, Dr. Emily C. Marcinowski, Dedrick Curtis, Amethyst Royal, Christina Wade-Giampanis, and Dr. Bernhard Völkl for data collection, helpful comments, and discussion in the preparation of this manuscript. Observations with and manipulations of our subjects were made in accordance with the Institutional Care and Use Committee (IACUC) guidelines at the University of North Carolina at the Greensboro, North Carolina, USA. 


\section{References}

396

397

398

399

400

401

402

403

404

405

406

407

408

409

410

411

412

413

414

415

416

417

418

419

420

421

422

423

424

425

426

427

428

429

430

431

432
Bailoo, J. D., Jordan, R. L., Garza, X. J., \& Tyler, A. N. (2013). Brief and long periods of maternal separation affect maternal behavior and offspring behavioral development in C57BL/6 mice. Developmental Psychobiology, 56(4), 674-685. http://doi.org/10.1002/dev.21135

Blaszczyk, J., \& Tajchert, K. (1996). Sex and strain differences of acoustic startle reaction development in adolescent albino Wistar and hooded rats. Acta Neurobiologiae Experimentalis, 56(4), 919-925. Retrieved from http://www.ncbi.nlm.nih.gov/pubmed/9033127

Braff, D. L., Geyer, M. A., \& Swerdlow, N. R. (2001). Human studies of prepulse inhibition of startle: Normal subjects, patient groups, and pharmacological studies. Psychopharmacology, 156(2-3), 234-258. http://doi.org/10.1007/s002130100810

Branchi, I., \& Cirulli, F. (2014). Early experiences: building up the tools to face the challenges of adult life. Developmental Psychobiology, 56(8), 1661-1674. Retrieved from http://www.ncbi.nlm.nih.gov/pubmed/24986379

Caldji, C., Francis, D., Sharma, S., Plotsky, P. M., \& Meaney, M. J. (2000). The effects of early rearing environment on the development of GABAA and central benzodiazepine receptor levels and novelty-induced fearfulness in the rat. Neuropsychopharmacology: Official Publication of the American College of Neuropsychopharmacology, 22(3), 219-29. Retrieved from http://www.ncbi.nlm.nih.gov/pubmed/10693149

Champagne, D. L., Bagot, R. C., van Hasselt, F., Ramakers, G., Meaney, M. J., de Kloet, E. R., ... Krugers, H. (2008). Maternal care and hippocampal plasticity: evidence for experiencedependent structural plasticity, altered synaptic functioning, and differential responsiveness to glucocorticoids and stress. The Journal of Neuroscience : The Official Journal of the Society for Neuroscience, 28(23), 6037-45. http://doi.org/10.1523/JNEUROSCI.052608.2008

de Kloet, E. R., Joëls, M., \& Holsboer, F. (2005). Stress and the brain: from adaptation to disease. Nature Reviews. Neuroscience, 6(6), 463-75. Retrieved from http://www.ncbi.nlm.nih.gov/pubmed/15891777

Ellenbroek, B. A., Geyer, M. A., \& Cools, A. R. (1995). The behavior of APO-SUS rats in animal models with construct validity for schizophrenia. The Journal of Neuroscience, 15(11), 7604-7611.

Geyer, M. A., Krebs-Thomson, K., Braff, D. L., \& Swerdlow, N. R. (2001). Pharmacological studies of prepulse inhibition models of sensorimotor gating deficits in schizophrenia: A decade in review. Psychopharmacology (Vol. 156). http://doi.org/10.1007/s002130100811

Ison, J. R., \& Hoffman, H. S. (1983). Reflex modification in the domain of startle: II. The anomalous history of a robust and ubiquitous phenomenon. Psychological Bulletin, 94(1), 3-17. http://doi.org/10.1037/0033-2909.94.1.3 
Kaffman, A., \& Meaney, M. J. (2007). Neurodevelopmental sequelae of postnatal maternal care in rodents: Clinical and research implications of molecular insights. Journal of Child Psychology and Psychiatry and Allied Disciplines, 48(3-4), 224-244. http://doi.org/10.1111/j.1469-7610.2007.01730.x

Lehmann, J., \& Feldon, J. (2000). Long-term biobehavioral effects of maternal separation in the rat: consistent or confusing? Reviews in the Neurosciences, 11(4), 383-408. Retrieved from http://www.ncbi.nlm.nih.gov/pubmed/11065281

Levine, S. (2002). Enduring Effects of Early Experience on Adult Behavior. In D. W. Plaff, A. P. Arnold, A. M. Etgen, S. E. Fahrbach, \& R. T. Rubin (Eds.), Hormones, Brain and Behavior, Volume 4 (pp. 535-542). New York, NY: Academic Press. Retrieved from https://books.google.com/books?id=6GgHpQdk8vYC\&pgis=1

Liu, D., Diorio, J., Tannenbaum, B., Caldji, C., Francis, D., Freedman, A., ... Meaney, M. J. (1997). Maternal care, hippocampal glucocorticoid receptors, and hypothalamic-pituitaryadrenal responses to stress. Science (New York, N.Y.), 277(5332), 1659-62. Retrieved from http://www.ncbi.nlm.nih.gov/pubmed/9287218

Macrí, S., Mason, G. J., \& Würbel, H. (2004). Dissociation in the effects of neonatal maternal separations on maternal care and the offspring's HPA and fear responses in rats. The European Journal of Neuroscience, 20(4), 1017-1024. http://doi.org/10.1111/j.14609568.2004.03541.x

Macrì, S., \& Würbel, H. (2006). Developmental plasticity of HPA and fear responses in rats: a critical review of the maternal mediation hypothesis. Hormones and Behavior, 50(5), 667680. http://doi.org/10.1016/j.yhbeh.2006.06.015

Matsumoto, Y., Niwa, M., Mouri, A., Ozaki, N., Nabeshima, T., Matsumoto, Y., ... Nabeshima, T. (2011). Vulnerability in early life to changes in the rearing environment plays a crucial role in the aetiopathology of psychiatric disorders. Japanese Journal of Neuropsychopharmacology, 14(4), 459-477. http://doi.org/10.1017/S1461145710001239

McEwen, B. S. (2003). Mood disorders and allostatic load. Biological Psychiatry, 54(3), 200207. Retrieved from http://www.sciencedirect.com/science/article/pii/S000632230300177X

Meaney, M. J. (2001). Maternal Care, Gene Expression, and the Transmission of Individual Differences in Stress Reactivity across Generations. Annual Review of Neuroscience, 24, 1161-1192.

Millstein, R. A., \& Holmes, A. (2007). Effects of repeated maternal separation on anxiety- and depression-related phenotypes in different mouse strains. Neuroscience and Biobehavioral Reviews, 31(1), 3-17. Retrieved from http://www.ncbi.nlm.nih.gov/pubmed/16950513

Millstein, R. A., Ralph, R. J., Yang, R. J., \& Holmes, A. (2006). Effects of repeated maternal separation on prepulse inhibition of startle across inbred mouse strains. Genes, Brain, and Behavior, 5(4), 346-354. Retrieved from http://www.ncbi.nlm.nih.gov/pubmed/16716204

Paylor, R., \& Crawley, J. N. (1997). Inbred strain differences in prepulse inhibition of the mouse startle response. Psychopharmacology, 132(2), 169-180. Retrieved from 
http://www.ncbi.nlm.nih.gov/pubmed/9266614

473

474

475

476

477

478

479

480

481

482

483

484

485

486

487

488

489

490

491

492

493

494

495

496

497

498

499

500

501

502

503

Pryce, C. R., Rüedi-Bettschen, D., Dettling, A. C., \& Feldon, J. (2002). Early life stress: longterm physiological impact in rodents and primates. News in Physiological Sciences, 17 , 150-155. http://doi.org/DOI 10.1152/nips.01367.2001

Schmidt, M. V. (2011). Animal models for depression and the mismatch hypothesis of disease. Psychoneuroendocrinology, 36(3), 330-338. Retrieved from http://www.sciencedirect.com/science/article/pii/S0306453010001551

Shoji, H., \& Kato, K. (2006). Maternal behavior of primiparous females in inbred strains of mice: a detailed descriptive analysis. Physiology \& Behavior, 89(3), 320-328. Retrieved from http://www.sciencedirect.com/science/article/pii/S0031938406002691

Smotherman, W. P., \& Bell, R. (1980). Maternal mediation of early experience. In R. W. Bell \& W. P. Smotherman (Eds.), Maternal Influences and Early Behavior (First, pp. 201-210). Spectrum Publications. Retrieved from https://books.google.com/books?id=IgmpNzaT3NkC\&pgis=1

Stern, J. M., \& Johnson, S. K. (1989). Perioral somatosensory determinants of nursing behavior in Norway rats (Rattus norvegicus). Journal of Comparative Psychology (Washington, D.C. : 1983), 103(3), 269-80. Retrieved from http://www.ncbi.nlm.nih.gov/pubmed/2776423

Swerdlow, N. R., Weber, M., Qu, Y., Light, G. A., \& Braff, D. L. (2008). Realistic expectations of prepulse inhibition in translational models for schizophrenia research.

Psychopharmacology (Vol. 199). http://doi.org/10.1007/s00213-008-1072-4

Varty, G. B., Braff, D. L., \& Geyer, M. A. (1999). Is there a critical developmental 'window' for isolation rearing-induced changes in prepulse inhibition of the acoustic startle response? Behavioural Brain Research, 100(1-2), 177-183.

Varty, G. B., \& Geyer, M. A. (1998). Effects of isolation rearing on startle reactivity, habituation, and prepulse inhibition in male Lewis, Sprague-Dawley, and Fischer F344 rats. Behavioral Neuroscience, 112(6), 1450-1457. http://doi.org/10.1037/0735-7044.112.6.1450

Weiss, I. C., Domeney, A. M., Moreau, J. L., Russig, H., \& Feldon, J. (2001). Dissociation between the effects of pre-weaning and/or post-weaning social isolation on prepulse inhibition and latent inhibition in adult Sprague-Dawley rats. Behavioural Brain Research, 121(1-2), 207-218. http://doi.org/10.1016/S0166-4328(01)00166-8 
5

6

7

8

504 Tables

505

506 Table 1

507

508 Total number of subjects used divided by pre-weaning group and post-weaning housing 509 condition.

510

511

\begin{tabular}{|c|c|c|c|c|c|}
\hline & \multicolumn{2}{|c|}{ Socially-housed } & \multicolumn{2}{|c|}{ Isolation-housed } & \multirow{2}{*}{ Total } \\
\hline & Male & Female & Male & Female & \\
\hline AFR & 9 & 12 & 5 & 10 & 36 \\
\hline EH & 11 & 13 & 3 & 3 & 30 \\
\hline MS & 11 & 11 & 14 & 10 & 46 \\
\hline MPS & 11 & 12 & 6 & 11 & 40 \\
\hline Total & 42 & 48 & 28 & 34 & 152 \\
\hline
\end{tabular}




\section{Figures}

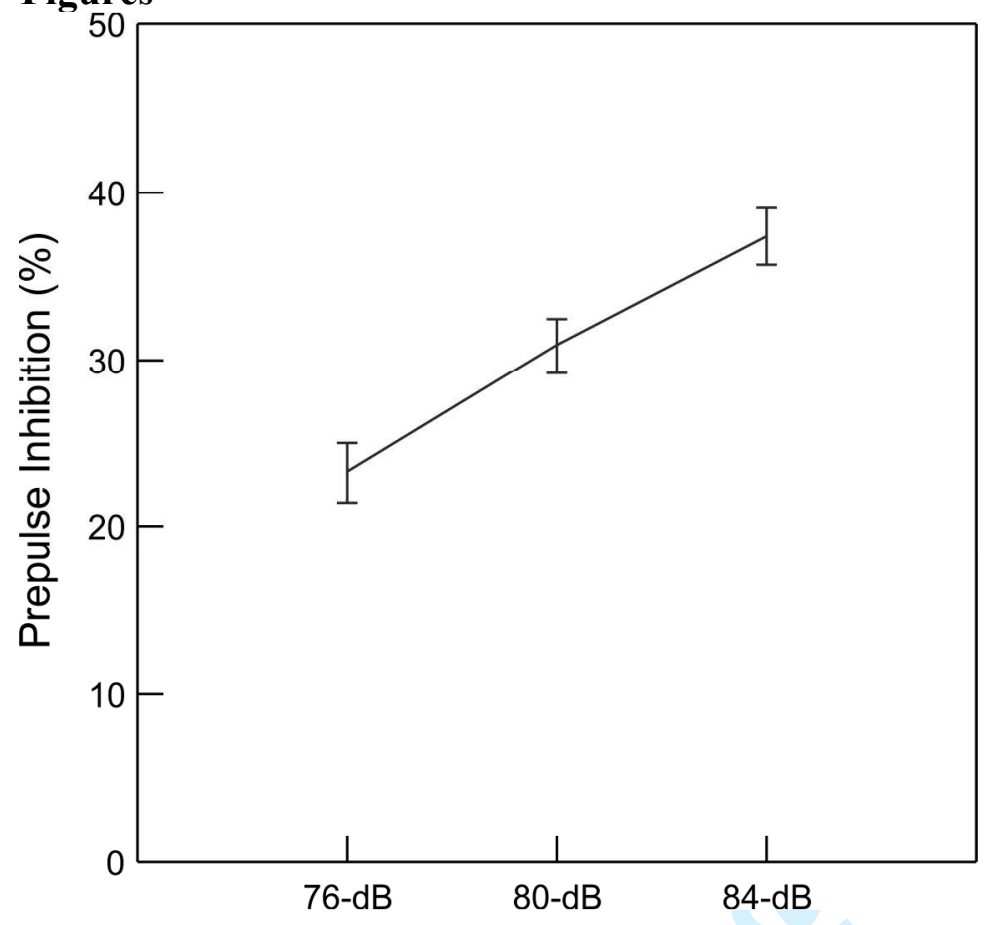

Figure 1. Differences in prepulse inhibition (\%) as a consequence of prepulse level (dB).

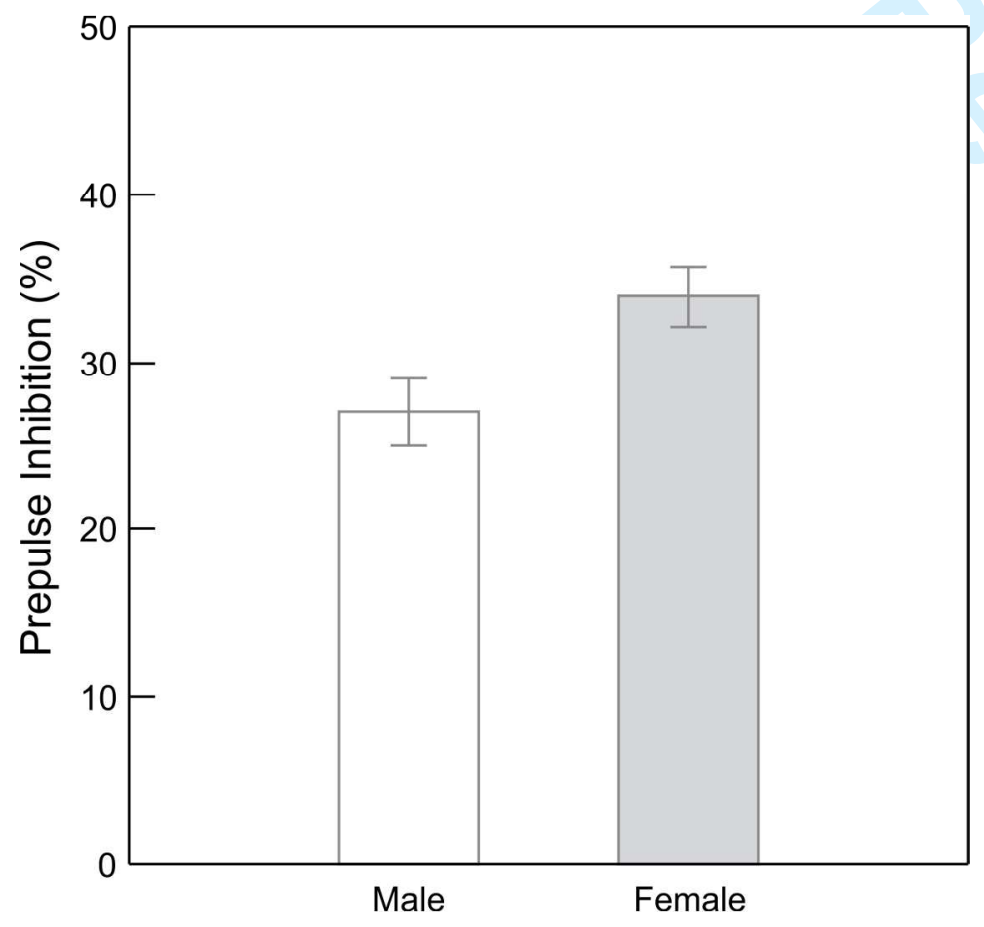

519 Figure 2. Differences in prepulse inhibition (\%) as a consequence of sex. 520 
526

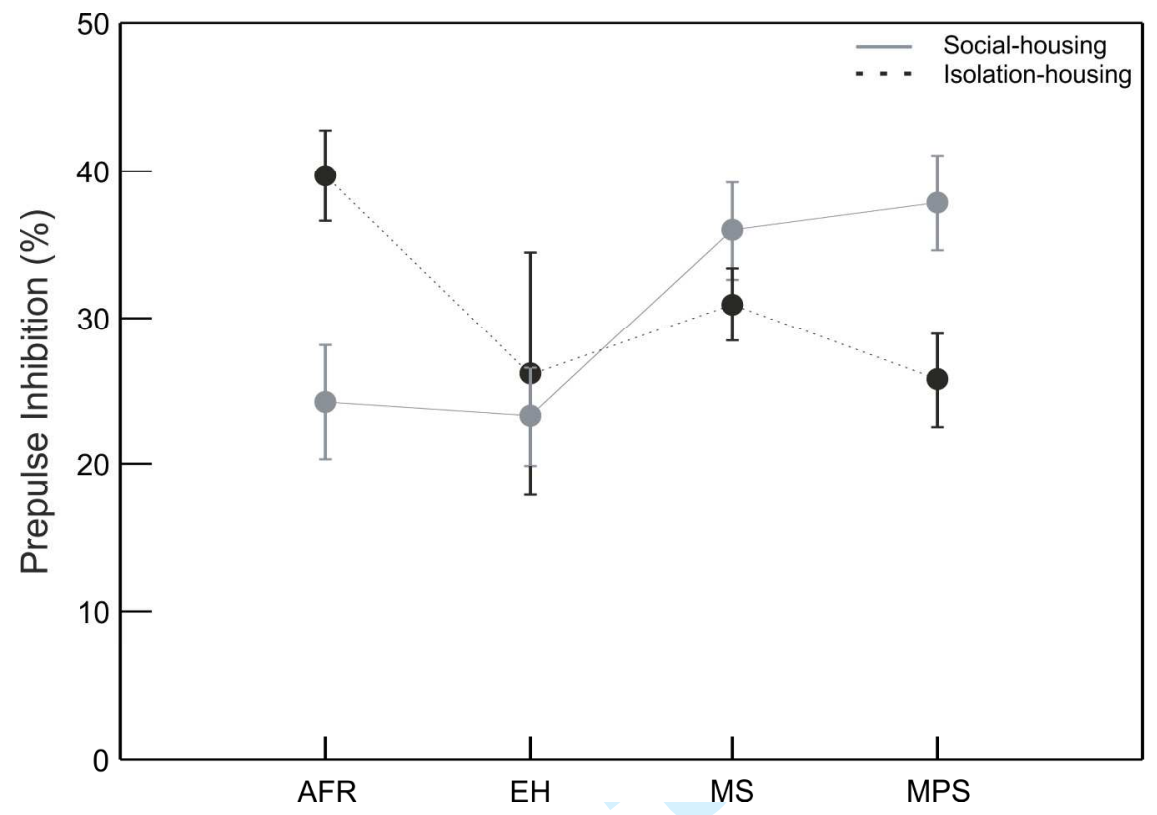

Figure 3. Differences in prepulse inhibition (\%) as a consequence of pre-weaning group and post-weaning housing condition.

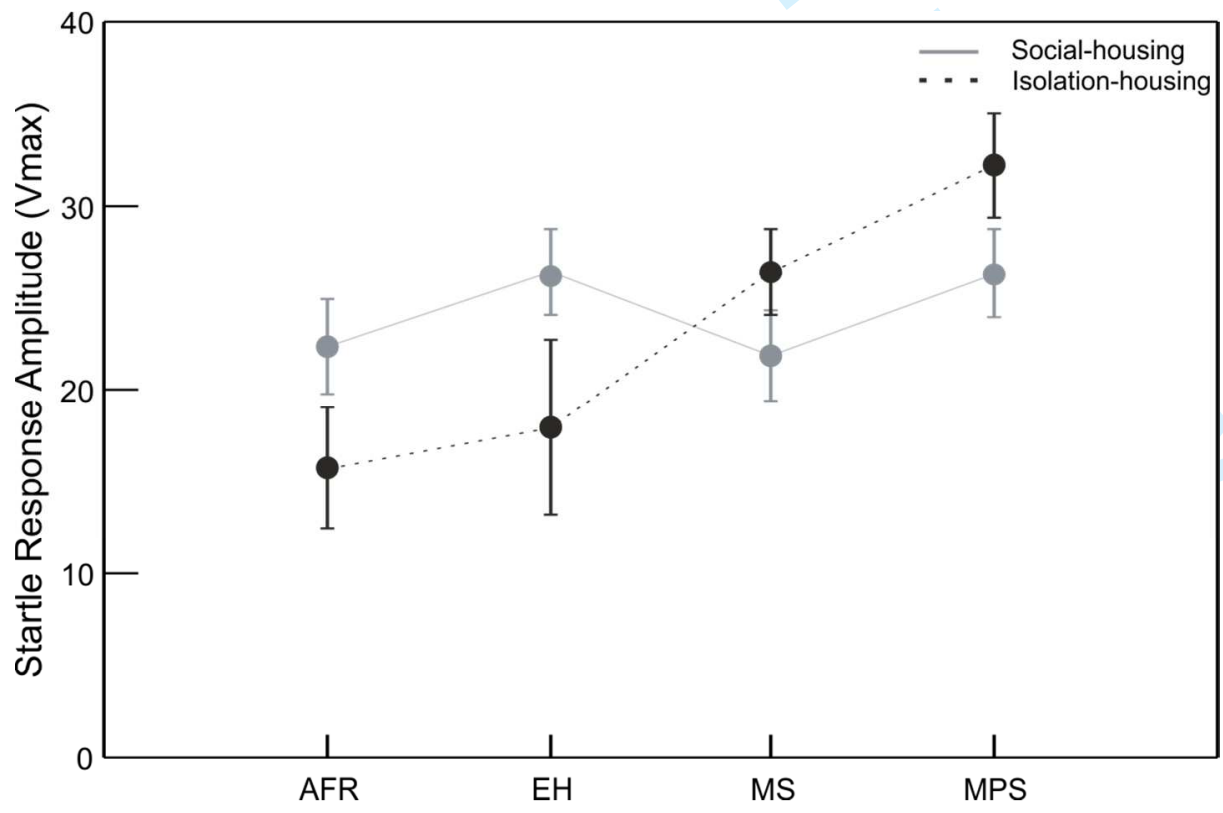

Figure 4. Differences in baseline startle amplitude $(68-\mathrm{dB})$ as a consequence of pre-weaning group and post-weaning housing condition. 


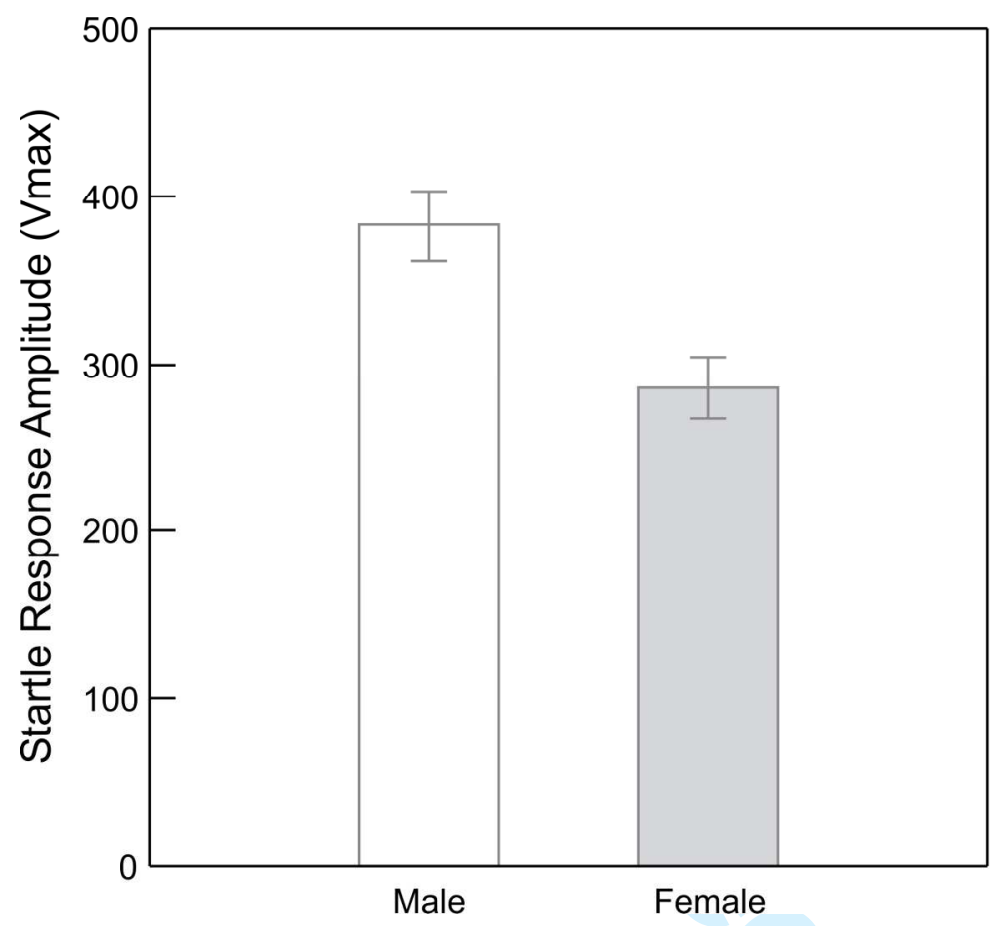

\section{4} 535 536

Figure 5. Differences in acoustic startle amplitude (120-dB) as a consequence of sex.

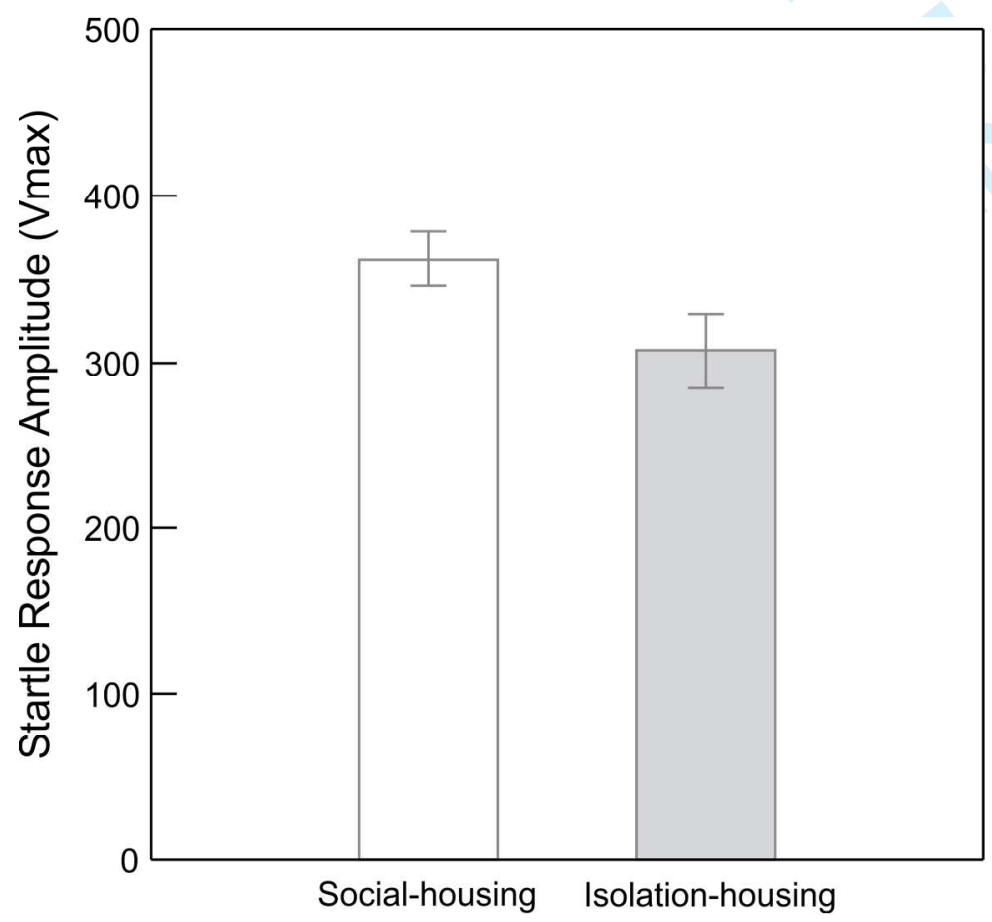

Figure 6. Differences in acoustic startle amplitude (120-dB) as a consequence of post-weaning housing condition. 